\title{
Representing the Model of Impedance Controlled Robot Interaction with Feedback Delay in Polytopic LPV Form: TP Model Transformation based Approach
}

\author{
Péter Galambos, Péter Baranyi
}

Computer and Automation Research Institute, Hungarian Academy of Sciences Kende u. 13-17. H-1111 Budapest, Hungary

\{galambos,baranyi\}@sztaki.hu

\begin{abstract}
The aim of this paper is to transform the model of the impedance controlled robot interaction with feedback delay to a Tensor Product (TP) type polytopic LPV model whereupon Linear Matrix Inequality (LMI) based control design can be immediately executed. The paper proves that the impedance model can be exactly represented by a finite element TP type polytopic model under certain constrains. The paper also determines various further TP models with different advantages for control design. First, it derives the exact Higher Order Singular Value Decomposition (HOSVD) based canonical form, then it performs complexity trade-off to yield a model with less number of components but rather effective for LMI design. Then the paper presents various different types of convex TP model representations based on the non-exact model in order to investigate how convex hull manipulation can be performed on the model. Finally the presented models are analyzed to validate the accuracy of the transformation and the resulting TP type polytopic LPV models. The paper concludes that these prepared models are ready for convex hull manipulation and LMI based control design.
\end{abstract}

Keywords: LPV/qLPV modeling; impedance conrol; complience control; time delay; telemanipulation; haptics

\section{Introduction}

The literature of modern control theory shows that the representation of a given plant has considerable effect on the usability of the proper controller design method and on the achievable control performance. For instance, in the case of the qLPV state-space model given in a polytop representation and the LMI (Linear Matrix Inequality) based design techniques, we can observe that the disposition layout of the system matrix elements at the very beginning modeling phase already determines 
the set of achievable control performance. Furthermore, the resulting controller really depends on the applied LMIs, which is why the majority of the related literature discusses how to manipulate LMIs in order to optimize for multi-objective control performance. At the same time, one of the key trends in modern control - $\mathcal{H}_{\infty}$ based methodologies - bases the optimization of the required control constraints on integrating weighting functions into the system model before determining the polytopic representation and constructing the LMI-based synthesis.

Nevertheless, it was not emphasized as much that the LMIs are very sensitive for the polytop structure. Since the LMI in that sense can be considered as a non-linear transformation, a little modification of the convex hull may lead to considerable deviation of the resulting controller. Therefore, one may raise the question whether the convex hull manipulation plays an important role in the optimization of the control performance. Actually, this was one of the key motivations to develop the TP model transformation, which is readily capable of manipulating the convex hull of the convex polytop representation of a given model. The TP model transformation is actually a numerical representation of the HOSVD of given functions. It becomes a control design tool when it is executed on matrix functions, where the matrix function actually represents the non linear system matrix of a given LPV/qLPV model. In this general case the TP model transformation can be viewed as a TP type polytop decomposition technique having various advantages for complexity tradeoff and convex hull manipulation, all relying on the power of the HOSVD. The key idea and further investigations about the utilization of TP model transformation is presented in papers [1, 2, 3]. Some further examples for control design oriented utilization of TP model transformation are in [4, 5, 6, 7, 8, 9, 10]. Authors Chumalee et al., Rangajeeva et al., Gai et al., Sun et al. and Qin et al. introduce TP model transformation based novel approaches in avionics related control problems $[11,12$, $13,14,15]$, thus leading to pioneering conceptual frameworks. In [16] Precup et al. introduced novel application-oriented TP models for the automatic transmission system of vehicles.

Paper [17] discusses the importance of the convex hull manipulation in the polytop representation based control design and how further improvements on the resulting control performance can be achieved. The paper also concludes that this manipulation results in a kind of relaxation of the conservativeness of the design. Based on this paper, Gróf at al. [18] deeply investigate an example how the convex hull manipulation influences the effectiveness of the LMIs, or even more how the improper selection of the convex hull may lead to infeasible LMIs. With this investigation, she has shown that the manipulation of the convex hull is as important as the selection of the LMIs to reach the best control performance.

In this paper, we examine two types of manipulation techniques. One type performs complexity trade-off on the number of the LTI vertex models, while the other focuses on the manipulation of the convex hull. Thus, we create the HOSVD based canonical form of the impedance model with approximation trade-off and generate different convex hulls using different convex transformation satisfying various constraints. 
The paper is organized as follows: Section 2 introduces definitions related to TP model transformation. Section 3 defines the equation of motion of the investigated delayed dynamical system and specifies the required properties of the expected model form. In section 4, the properties of the resulted HOSVD-based canonical form are discussed and a trade-off is performed between the complexity and the accuracy of the TP model. Section 5 introduces the model with different types of convex hulls, and section 6 investigates the accuracy of the resulted convex TP model considering constant and varying time delay. The last section concludes the paper.

\section{Basic concepts}

The mathematical background of the TP model transformation and TP model transformation based LMI controller design was introduced and elaborated in $[1,2,3]$. Let us recall some of the related theorems and definitions:

Definition 1 (qLPV model): Consider the Linear Parameter Varying State Space model:

$$
\begin{aligned}
\dot{\mathbf{x}}(t) & =\mathbf{A}(\mathbf{p}(t)) \mathbf{x}(t)+\mathbf{B}(\mathbf{p}(t)) \mathbf{u}(t) \\
\mathbf{y}(t) & =\mathbf{C}(\mathbf{p}(t)) \mathbf{x}(t)+\mathbf{D}(\mathbf{p}(t)) \mathbf{u}(t),
\end{aligned}
$$

with input $\mathbf{u}(t) \in \mathbb{R}^{m}$, output $\mathbf{y}(t) \in \mathbb{R}^{l}$ and state vector $\mathbf{x}(t) \in \mathbb{R}^{k}$. The system matrix

$$
\mathbf{S}(\mathbf{p}(t))=\left(\begin{array}{ll}
\mathbf{A}(\mathbf{p}(t)) & \mathbf{B}(\mathbf{p}(t)) \\
\mathbf{C}(\mathbf{p}(t)) & \mathbf{D}(\mathbf{p}(t))
\end{array}\right)
$$

is a parameter-varying object, where $\mathbf{p}(t) \in \Omega$ is a time varying $N$-dimensional parameter vector, and $\Omega=\left[a_{1}, b_{1}\right] \times\left[a_{2}, b_{2}\right] \times \ldots \times\left[a_{N}, b_{N}\right] \in \mathbb{R}^{N}$ is a closed hypercube. $\mathbf{p}(t)$ can also include some elements of $\mathbf{x}(t)$. In this case, (2) is referred to as a quasi $L P V(q L P V)$ model. This type of model is considered to belong to the class of non-linear models. The size of the system matrix $\mathbf{S}(\mathbf{p}(t))$ is $(k+l) \times(k+m)$.

A wide class of LMI based control design techniques are available for convex polytopic model representations; thus the finite element convex polytopic form of (1) is defined as:

Definition 2 (Finite element polytopic model):

$$
\mathbf{S}(\mathbf{p}(t))=\sum_{r=1}^{R} w_{r}(\mathbf{p}(t)) \mathbf{S}_{r} .
$$

where $\mathbf{p}(t) \in \Omega . \mathbf{S}(\mathbf{p}(t))$ is given for any parameter vector $\mathbf{p}(t)$ as the parameter varying combinations of LTI system matrices $\mathbf{S}_{r} \in \mathbb{R}^{(k+l) \times(k+m)}$ called LTI vertex 
systems. The combination is defined by weighting functions $w_{r}(\mathbf{p}(t)) \in[0,1]$. The term finite means that $R$ is bounded.

Definition 3 (Finite element TP type polytopic model): $\mathbf{S}(\mathbf{p}(t))$ in (3) is given for any parameter as the parameter-varying combination of LTI system matrices $\mathbf{S}_{r} \in$ $\mathbb{R}^{(k+l) \times(k+m)}$.

$$
\mathbf{S}(\mathbf{p}(t))=\sum_{i_{1}=1}^{I_{1}} \sum_{i_{2}=1}^{I_{2}} . . \sum_{i_{N}=1}^{I_{N}} \prod_{n=1}^{N} w_{n, i_{n}}\left(p_{n}(t)\right) \mathbf{S}_{i_{1}, i_{2}, . ., i_{N}},
$$

applying the compact notation based on tensor algebra (Lathauwer's work [19]) one has:

$$
\mathbf{S}(\mathbf{p}(t))=S \underset{n=1}{\stackrel{N}{\bigotimes}} \mathbf{w}_{n}\left(p_{n}(t)\right)
$$

where the $(N+2)$ dimensional coefficient tensor $\mathbf{S} \in \mathbb{R}^{I_{1} \times I_{2} \times \cdots \times I_{n} \times(m+k) \times(m+k)}$ is constructed from the LTI vertex systems $\mathbf{S}_{i_{1}, i_{2}, \ldots, i_{N}}(5)$ and the row vector $\mathbf{w}_{n}\left(p_{n}(t)\right)$ contains univariate and continuous weighting functions $w_{n, i_{n}}\left(p_{n}(t)\right),\left(i_{n}=1 \ldots I_{N}\right)$.

Remark 1 : TP model (5) is a special class of polytopic models (3), where the weighting functions are decomposed to a Tensor Product of univariate functions.

Definition 4 (TP model transformation): TP model transformation is a numerical method that transforms qLPV models given in the form of (1) to the form of (5), so that a large class of LMI based control design techniques can be applied to the resulting model. Detailed description of TP model transformation and application examples can be found in [1]. The TP model transformation gives a trade-off between the accuracy of the resulting model and the number of required vertexes for the LMI control design. The TP model transformation is also capable of providing a convex hull manipulation tool during execution. For further details please read papers [17, 18].

Definition 5 (HOSVD-based canonical form of qLPV models): The direct result of the TP model transformation when neither complexity trade-off nor convex hull manipulation is done is the numerical reconstruction of the HOSVD of a given function. It is like the HOSVD of tensors, but for functions where instead of singular matrices we have singular functions in an orthonormal structure, and the core tensor contains the higher order singular values. In the case of a system where matrix functions are used, the HOSVD canonical form has the same structure; the only difference is that the core tensor contains the system vertices assigned to the higher order singular values. For further details please be referred to papers [20, 21].

Definition 6 (Convex TP model): The TP model is convex if the weighting functions satisfy the following criteria:

$$
\forall n, i, p_{n}(t): w_{n, i}\left(p_{n}(t)\right) \in[0,1]
$$




$$
\forall n, p_{n}(t): \sum_{i=1}^{I_{n}} w_{n, i}\left(p_{n}(t)\right)=1
$$

Different convex hulls for TP type polytopic qLPV models can be defined. Some of the basic types are defined as follows:

Definition 7 (SN type TP function): The convex TP function is SN (Sum Normalized) if the sum of the weighting functions for all $x \in \Omega$ is 1 .

Definition 8 (NN type TP function): The convex TP function is NN (Non-Negative) if the values of the weighting functions for all $x \in \Omega$ are non-negative.

Definition 9 (NO/CNO, NOrmal type TP function): The convex TP function is a NO (Normal) type model if its $w(p)$ weighting functions are Normal, that is, if it satisfies (6) and (7), and the largest value of all weighting functions is 1. Also, it is CNO (close to normal), if it is satisfies (6) and (7) and the largest value of all weighting functions is 1 or close to 1 .

Definition 10 (IRNO, Inverted and Relaxed NOrmal type TP function): The TP function is IRNO type if the smallest values of all weighting functions are 0, and the largest values of all weighting functions are the same.

\section{Specification of the modeling problem}

Since the extensive work of Hogan [22, 23, 24], wherein the concept of impedance control and its application was formulated, this control strategy has become one of the key technologies of modern robot control. Haptic rendering is a special area of robotics where the haptic device and the virtual environment together forms an impedance controlled interaction structure where time delays have an unfavorable effect on the stability of the system. A series of papers from DLR's researchers investigate the stability of haptic rendering from various aspects [25, 26, 27]. The present study focuses on the time delay that occurs in the control loop of the impedance controlled interaction.

In this paper, impedance model is understood as the dynamic relationship between the force and the resulted displacement. The impedance model is typically given by a virtual mass-spring-damper system. In the general case, a task-space impedance model can be described as

$$
\mathbf{M} \ddot{\mathbf{x}}+\mathbf{B} \dot{\mathbf{x}}+\mathbf{K} \mathbf{x}+\mathbf{C}(\dot{\mathbf{x}}, \mathbf{x})=\mathbf{F},
$$

where $\mathbf{x}$ denotes the Cartesian task space coordinates, $\mathbf{M}, \mathbf{B}$ and $\mathbf{K}$ are symmetric, positive-definite matrices describing the inertial, damping and stiffness parameters 
respectively, and $\mathbf{C}$ contains other non-linear terms of the impedance model, while $\mathbf{F}$ denotes the external forces.

In many cases, the end effector path is prescribed and the displacement results from the impedance model is added to the predefined path. In this way the robot motion becomes compliant.

\subsection{Equation of the impedance controlled actuation with feedback delay}

Since this paper deals with a generic abstraction, a single degree of freedom model will be discussed, but the results can be extended to multidimensional cases. Consider the mechanical system depicted by Figure 1(a) as a simplified model of the impedance controlled robot interaction. Mass $m$ and viscous damping $b$ are virtual properties defining the desired dynamics of the manipulator, while $k$ denotes the stiffness of the robot's environment.

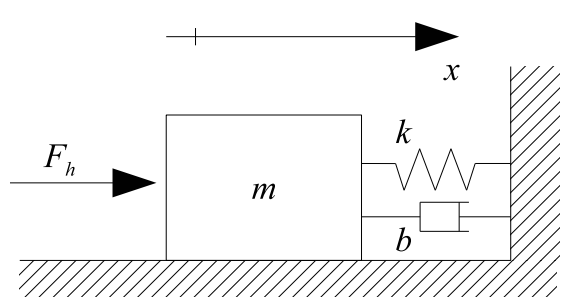

(a) Mass-Spring-Damper system as the simplified model of impedance controlled robot interaction.

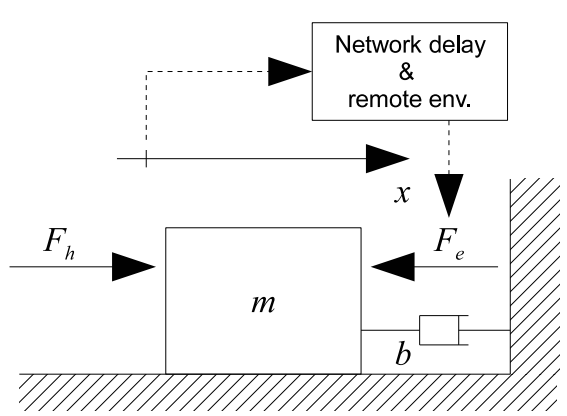

(b) Mass-Spring-Damper model where the effect of the spring is delayed

Figure 1

Introducing the time-delay in the measurement of the interaction force between the robot and its environment.

Virtual parameters have to be chosen according to the accuracy $\leftrightarrow$ robustness tradeoff [28]: The lower mass and damping result in faster and more accurate tracking with less robustness against feedback delay, and vice versa.

The equation of motion of this system is as follows:

$$
\ddot{x}(t)=\frac{F_{h}(t)}{m}-\frac{b}{m} \dot{x}(t)-\frac{F_{e}(t)}{m}
$$

Introducing the time-delay $\tau$ in the interaction as the overall delay of the force 
monitoring due to the lag of the signal processing and/or network delays:

$$
\ddot{x}(t)=\frac{F_{h}(t)}{m}-\frac{b}{m} \dot{x}(t)-\frac{F_{e}(t-\tau(t))}{m}
$$

substituting the interaction force $\left(F_{e}\right)$ by the elastic force $(k x)$ in the formula as the simplest model of the environment, we get the following equation:

$$
\ddot{x}(t)=\frac{F_{h}(t)}{m}-\frac{b}{m} \dot{x}(t)-\frac{k}{m} x(t-\tau(t)) .
$$

One can see that the resulted equation represents a mass-spring-damper system where the elastic effect is delayed by $\tau(t)$. Figure 1(b) illustrates the resulted model.

\subsection{Specification of the expected qLPV reprezentation}

In this paper, we search for a representation of the investigated delayed dynamical system in TP type polytopic form (5), wherein the time delay $\tau$ becomes a parameter of the model and meets the following requirements:

$i$ Fulfills the specifications of HOSVD based canonical form by finding the minimum number of LTI components that represent the original system in polytopic structure (4).

ii Complexity trade-off capability by means of approximation.

iii Eligibility for LMI based multi-objective control design.

$i v$ The generated convex hull indirectly supports the feasibility of optimal control performance under the LMI based design concept.

\section{The HOSVD based canonical form}

In this section we utilize TP model transformation to determine the so called HOSVD based canonical form (Theorem 5) of the investigated model (11), which is a minimum and unique TP type polytopic representation. Since the investigated delayed model cannot be discretized by sampling in the first step of the TP model transformation, the discretized system tensor was determined based on the identification (see the paper [29]) of the model with multiple delay values. As the TP model transformation is a fully numerical method, the paper discusses a typical numerical example, wherein the following model parameters are considered: Mass $m=1 \mathrm{~kg}$, viscous damping $b=100 \mathrm{Ns} / \mathrm{m}$, Stiffness of the environment $k=2000 \mathrm{~N} / \mathrm{m}$, Delay interval $\tau=0 . .0 .07 \mathrm{~s}$. It is important to note that the main properties of the polytop structure are not influenced significantly by the model parameters in a wide range with practical relevancy. 


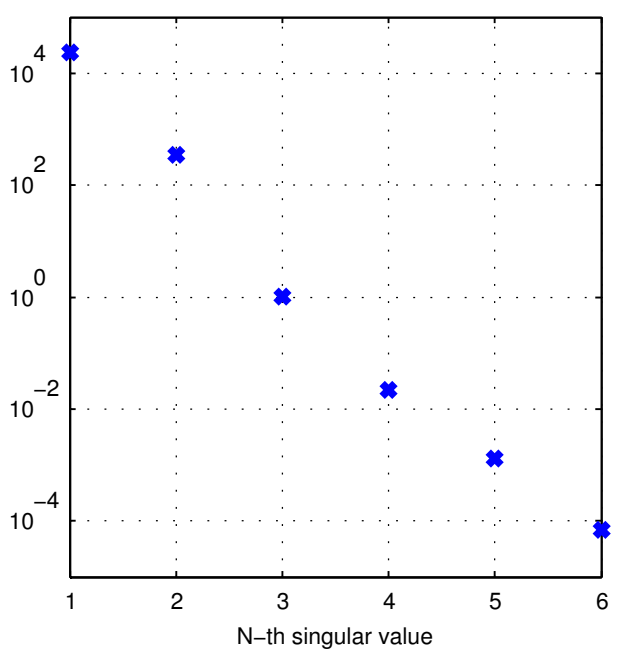

Figure 2

Singular values of the HOSVD based canonical form

\subsection{Components and structure of the exact HOSVD based canoni- cal form}

After the execution of the TP model transformation on the impedance model, we get the minimum size qLPV representation composed of 6 LTI vertex models since the HOSVD leads to 6 non-zero singular values in the second step of the TP model transformation. Singular values are as follow: $\sigma_{1}=2.3414 \times 10^{4}, \sigma_{2}=3.5305 \times 10^{2}$, $\sigma_{3}=1.0331, \sigma_{4}=2.2164 \times 10^{-2}, \sigma_{5}=1.2964 \times 10^{-3}, \sigma_{6}=6.9808 \times 10^{-5}$. Note again that the different model parameters have no substantial effect on the resulted singular values, on the rank of the model or on the underlying polytopic structure, so this example properly shows the uniqueness of the representation. The consecutive singular values decrease exponentially by a factor of two orders of magnitude, which suggests a balanced contribution of vertices. Figure 2 displays the formation of the 6 singular values.

In the following, the components of the HOSVD based canonical form of the impedance model are introduced. The system is represented by the vertex models and the weighting functions. Let us partition the LTI vertices $\left(\mathbf{S}_{r}^{c a n}\right)$ as follows:

$$
\mathbf{S}_{r}^{c a n}=\left[\begin{array}{ll}
\mathbf{A} & \mathbf{B} \\
\mathbf{C} & \mathbf{D}
\end{array}\right]
$$

As the transformation results in $\mathbf{C}=\left[\begin{array}{ll}1 & 0\end{array}\right]^{T}$ and $\mathbf{D}=0$ for all $\tau \in \Omega$, only $\mathbf{A}$ and $\mathbf{B}$ are written in the list below: 


$$
\begin{aligned}
{[\mathbf{A B}]_{1}^{c a n} } & =\left[\begin{array}{ccc}
-1.1515 \times 10^{4} & -1.1896 \times 10^{4} & -6.3944 \\
1.1521 \times 10^{4} & 1.1890 \times 10^{4} & 6.3906
\end{array}\right] \\
{[\mathbf{A B}]_{2}^{\text {can }} } & =\left[\begin{array}{lll}
-1.8082 \times 10^{2} & 1.7522 \times 10^{2} & 3.0496 \\
1.7781 \times 10^{2} & -1.7209 \times 10^{2} & -3.0457
\end{array}\right] \\
{[\mathbf{A B}]_{3}^{c a n} } & =\left[\begin{array}{lll}
2.8404 \times 10^{-1} & -2.7537 \times 10^{-1} & -6.0693 \times 10^{-1} \\
2.9928 \times 10^{-1} & -2.9109 \times 10^{-1} & 6.0668 \times 10^{-1}
\end{array}\right] \\
{[\mathbf{A B}]_{4}^{c a n} } & =\left[\begin{array}{lll}
8.7316 \times 10^{-3} & -9.6607 \times 10^{-3} & 8.6616 \times 10^{-3} \\
8.7412 \times 10^{-3} & -9.6704 \times 10^{-3} & -8.7600 \times 10^{-3}
\end{array}\right] \\
{[\mathbf{A B}]_{5}^{\text {can }} } & =\left[\begin{array}{lll}
6.6570 \times 10^{-4} & 6.2594 \times 10^{-4} & 9.5164 \times 10^{-5} \\
6.6535 \times 10^{-4} & 6.2629 \times 10^{-4} & 3.9904 \times 10^{-5}
\end{array}\right] \\
{[\mathbf{A B}]_{6}^{\text {can }} } & =\left[\begin{array}{lll}
-2.5581 \times 10^{-6} & -2.5893 \times 10^{-6} & 4.9197 \times 10^{-5} \\
-2.5570 \times 10^{-6} & -2.5904 \times 10^{-6} & 4.9258 \times 10^{-5}
\end{array}\right]
\end{aligned}
$$

Figure 3 shows the weighting functions $\mathbf{w}(\tau)$ over the range of $\Omega$. The smoothness of the weighting functions shows that the applied reidentification method is stable along the investigated range of $\tau$. This means that the applied identification algorithm does not alternate between different local solutions (local minimums). It is worth mentioning that if the identification method is switching between different solutions, additional ranks could appear in the HOSVD canonical form. By neglecting the extra singular values, HOSVD is able to (smoothly) approximate the ruggedness in least-square sense in a way similar to how SVD can be used for noise filtering in digital signal processing [30]. However, if the fluctuation is large, such approximation should be applied with circumspection.

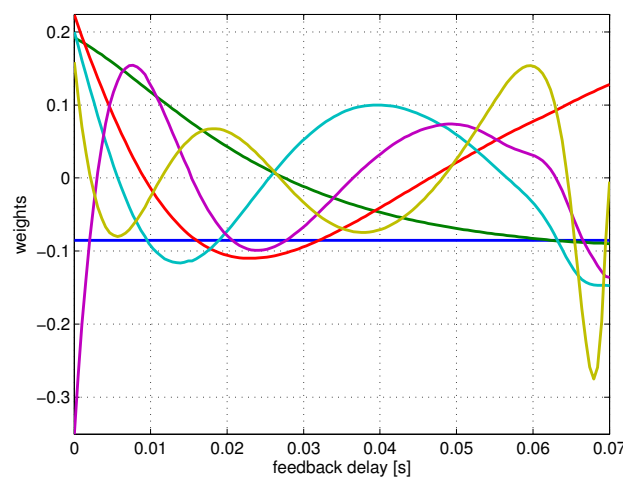

Figure 3

Weighting functions of the HOSVD based canonical form

\subsection{Executing trade-off by TP model transformation}

As was mentioned before, a trade-off can be determined between the complexity and the accuracy of the TP type polytopic model. The goal of this subsection is 
to reveal the correlation between the accuracy and the number of utilized vertices. Considering that the LMI based design process is very sensitive to the complexity of the polytopic model, it is very important to find the minimum complexity that reaches the accuracy threshold of the given engineering problem.

Even for the computational solutions of LMI toolbox for MATLAB that introduces high quality LMI solvers [31], the computational requirements explodes exponentially by the number of vertex models. Over a certain number of vertices, the LMI solvers may not be able to provide the solution. Considering that the significance of the vertex models decreases uniformly (Figure 2), there is no theoretically appealing point from where to cut the less significant vertices to reduce the complexity of the model. However, a systematically executed trade-off could help to find the reasonable complexity. The HOSVD based canonical form readily supports a kind of principal component analysis of the investigated dynamical system model. In this analysis, the model accuracy is measured by the modeling error $\epsilon_{r}$ defined as:

$$
\epsilon_{r}=\left\|\mathcal{S}^{D(\Omega, M)}-\mathcal{S}_{\text {Approx }_{r}}^{D(\Omega, M)}\right\|_{\mathcal{L}_{2}},
$$

where $\mathcal{S}^{D(\Omega, M)}$ can be computed using CHOSVD in the second step of the TP model transformation. $\mathcal{S}_{\text {Approx }}^{D(\Omega, M)}$ is computed analogously but considers only the vertex models according to the first $r$ singular values. Modeling errors result as follows:

$$
\begin{aligned}
& \epsilon_{1}=3.53 \times 10^{2} \leq 3.541 \times 10^{2} \\
& \epsilon_{2}=1.0333 \leq 1.0566 \\
& \epsilon_{3}=2.22 \times 10^{-2} \leq 2.35 \times 10^{-2} \\
& \epsilon_{4}=1.3 \times 10^{-3} \leq 1.4 \times 10^{-3} \\
& \epsilon_{5}=6.9808 \times 10^{-5} \leq 6.9808 \times 10^{-5} \\
& \epsilon_{6}=1.1272 \times 10^{-11} \approx 0 \text { (numerically zero) }
\end{aligned}
$$

As matrix $[\mathbf{A B}]_{r}$ contains element in the order of magnitude $10^{3}$, due to the definition of $\epsilon_{r}, \epsilon_{6}$ is much larger then $10^{-15}$, which is typically considered as numerically zero if all the matrix elements are in the range of $10^{1} . \epsilon_{r}$ is upper bounded by the sum of the singular values of the neglected vertices. The upper bounds are also displayed in the above list.

Figure 4(a) displays the modeling errors. One can see that the modeling error decreases between $\epsilon_{6}$ and $\epsilon_{5}$ much larger then in the case of the other reduction steps.

This measure describes the model accuracy only over the discrete delay values defined by $M$ and does not give information about the correctness between the discrete points that have been used in the first step of the TP model transformation. To follow a more extensive investigation and to ensure that the resulted TP model is not under-sampled, let us define the following measure: 


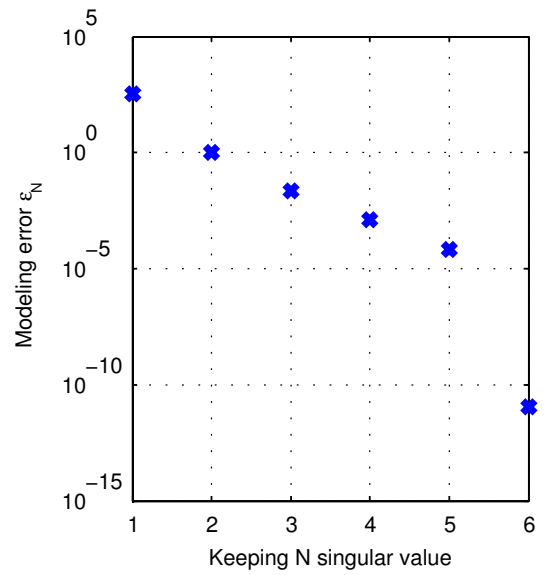

(a) Modelling error $\left(\epsilon_{r}\right)$ in function of dimensionality of HOSVD based canonical form

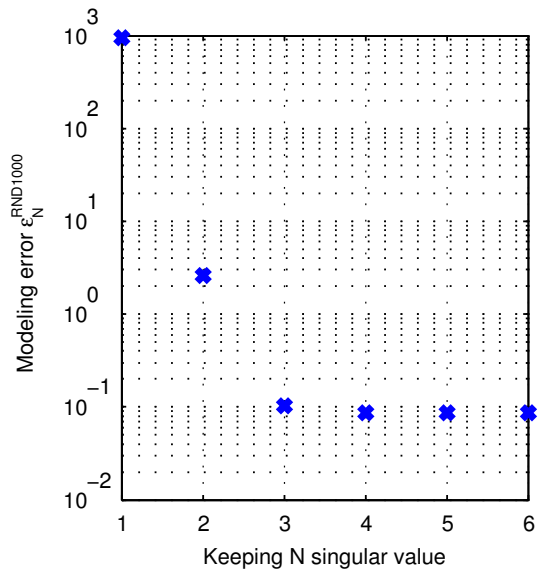

(b) Modelling error $\left(\epsilon_{r}^{R N D 1000}\right)$ in function of dimensionality of hosvd-based canonical form

Figure 4

Accuracy-complexity trade-off

\section{Definition $11\left(\epsilon_{r}^{\mathrm{RND} 1000}\right)$}

$$
\epsilon_{r}^{R N D 1000}=\left\|\mathcal{S}^{D\left(\Omega, M^{\prime}\right)}-\mathcal{S}_{\text {Approx }_{r}}^{D\left(\Omega, M^{\prime}\right)}\right\|_{\mathcal{L}_{2}},
$$

where $M^{\prime}$ denotes a discretization grid with 1000 randomly generated grid points over $\Omega$. Grid $M^{\prime}$ is not equidistant and $M^{\prime} \cap M=\emptyset$.

The measure $\epsilon_{r}^{\mathrm{RND} 1000}$ compares the reidentified and the approximated systems in 1000 randomly generated points considering the first $r$ vertices of the HOSVD based model. $\epsilon_{r}^{\mathrm{RND} 1000}$ shows the model accuracy better in real situations where arbitrary varying delays occur. The resulted $\epsilon_{r}^{\mathrm{RND} 1000} \mathrm{~s}$ are listed below:

$$
\begin{aligned}
\epsilon_{1}^{\mathrm{RND} 1000} & =9.6325 \times 10^{2} \\
\epsilon_{2}^{\mathrm{RND} 1000} & =2.7698 \\
\epsilon_{3}^{\mathrm{RND} 1000} & =1.2099 \times 10^{-1} \\
\epsilon_{4}^{\mathrm{RND} 1000} & =1.0519 \times 10^{-1} \\
\epsilon_{5}^{\mathrm{RND} 1000} & =1.0514 \times 10^{-1} \\
\epsilon_{6}^{\mathrm{RND} 1000} & =1.0514 \times 10^{-1}
\end{aligned}
$$

The values for $r=4, r=5, r=6$ are almost the same and start to increase only at 
$r=3$. Figure 4(b) displays the $\epsilon_{r}^{\mathrm{RND} 1000}$ data in logarithmic scale and the big step by $r=3$ is evident.

These results support the hypothesis that the number of non-zero singular values do not increase, even when the density of $M$ is increased without bounds. Thus, results show that the representation is minimal and exact. It can also be concluded that the applied discretization is not under-sampled, hence complexity reduction by neglecting the less significant vertices is well established. Beyond this pure numerical comparison, the dynamic accuracy of the TP model is also investigated in section 6.

\section{Different convex TP model representations}

As was already emphasized, LMIs are very sensitive to the shape of the convex hull that defines the polytopic qLPV representation together with the weighting functions. Different types of convex hulls of the delayed impedance model can be generated utilizing the hull manipulation capabilities of TP model transformation. For the sake of brevity, only the non-exact, CNO type convex model with 3 vertices is detailed here. Even using the most developed optimization strategies, it is not possible to generate NO type convex hulls. From the engineering aspect, this hypothesis can be accepted since the CNO type convex hulls fulfill the requirements of control synthesis.

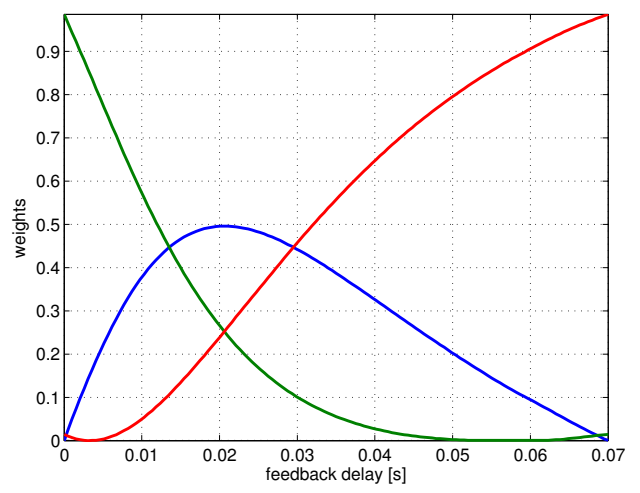

Figure 5

Weighting functions of CNO type convex hull of the reduced TP model with 3 vertices

$$
\begin{aligned}
{[\mathbf{A B}]_{1}^{\text {cnos }} } & =\left[\begin{array}{ccc}
9.7873 \times 10^{2} & 1.0209 \times 10^{3} & 8.6644 \times 10^{-1} \\
-9.7957 \times 10^{2} & -1.0200 \times 10^{3} & -8.6591 \times 10^{-1}
\end{array}\right] \\
{[\mathbf{A B}]_{2}^{\text {cno3 }} } & =\left[\begin{array}{ccc}
9.4943 \times 10^{2} & 1.0519 \times 10^{3} & 1.0101 \\
-9.5043 \times 10^{2} & -1.0509 \times 10^{3} & -1.0091
\end{array}\right] \\
{[\mathbf{A B}]_{3}^{\text {cno3 }} } & =\left[\begin{array}{ccc}
1.0005 \times 10^{3} & 9.9947 \times 10^{2} & 1.8247 \times 10^{-1} \\
-1.0007 \times 10^{3} & -9.9929 \times 10^{2} & -1.8254 \times 10^{-1}
\end{array}\right]
\end{aligned}
$$




\section{Analysis of the convex representation}

The goal of this section is to illustrate the dynamical accuracy of the different TP models. Here the model accuracy is investigated by means of the difference between the step response of the polytopic model and the original delayed model. Due to the limited extent of this paper, only a set of practically interesting validation cases have been included. The comparison is broken into two parts according to the constant delay and varying delay cases.

\subsection{Constant time-delay}

Firstly, the dynamic accuracy of the HOSVD based canonical forms of the delayed impedance model with different complexity is examined. Figure 6 shows the step responses of the compared models at an arbitrarily chosen constant delay value $(\tau=0.05567)$. As input signal, a $1 N$ force step was used at $0.1 \mathrm{~s}$ in the simulation. Subfigures 6/a-f shows the step responses of TP models with a different number of neglected less significant vertices. The time plots confirm the result of the modeling error analysis done in 4.2. As the values of $\epsilon_{r}^{\mathrm{RND} 1000}$ suggested, the TP models show similarly good accuracy with 6,5 and 4 vertices and the model accuracy begins to relapse with 3 vertices. A TP model with 2 and only 1 vertices cannot describe the dynamics of the original delayed system properly.

For the purpose of confidence, the same simulations have been executed on CNO type TP models with 5, 4 and 3 vertices (Figure 7). As was expected, the time plots show the same result as the HOSVD based canonical type with equivalent complexities.

We can conclude that the CNO type TP models with 5,4 and 3 vertices give very similar responses independently from the complexity. The convex hull of the investigated polytopic model cannot be formed with less then three vertices because the resulted domain of LPV models are not on the hyperline that can be defined by the convex combinations of two vertex systems. The results suggest that the CNO type convex TP model with 3 vertices provides sufficient accuracy for controller design purposes.

For the quantitative comparison, the $\mathcal{L}_{2}$ norm of the position error (the square root of sum of squares) and the maximum error is computed at four arbitrarily chosen $\tau$ values (neither of them are on the grid $M$ ) considering the $1 s$ long execution of the previously discussed simulation scenario. Results are displayed in Table 1. 


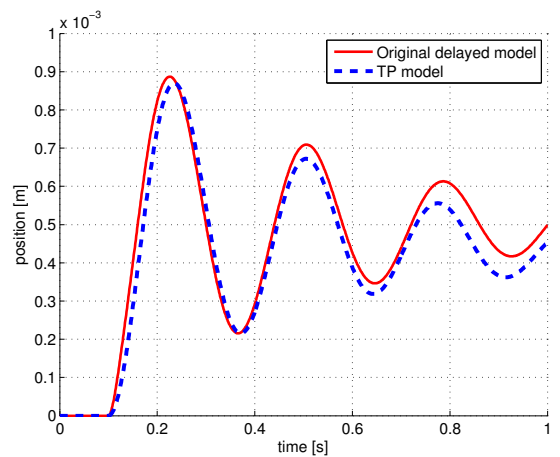

(a) Canonical form with 6 vertices (exact model)

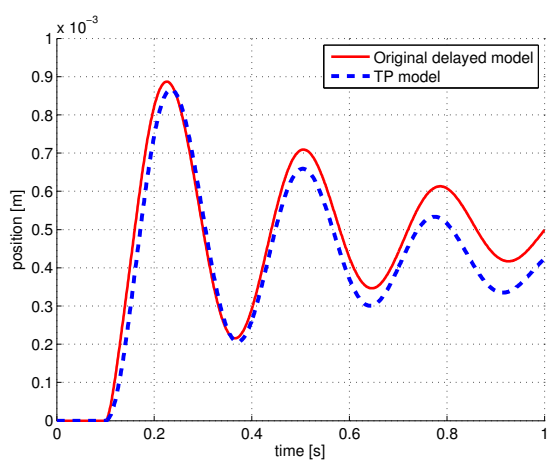

(c) Canonical form with 4 vertices

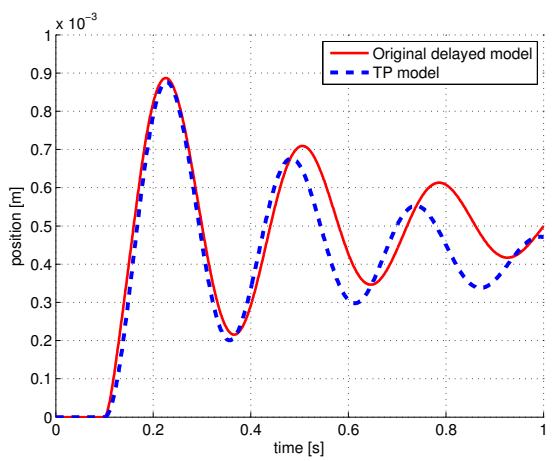

(e) Canonical form with 2 vertices

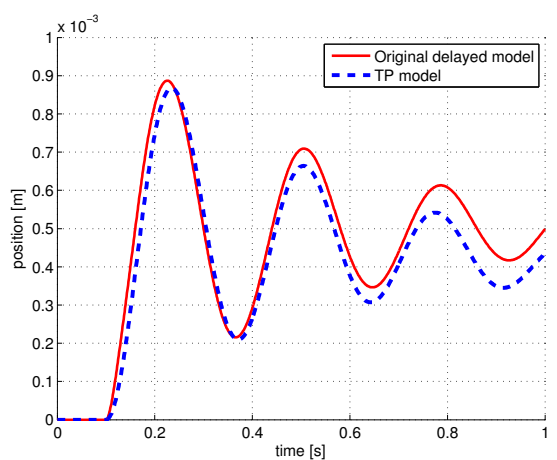

(b) Canonical form with 5 vertices

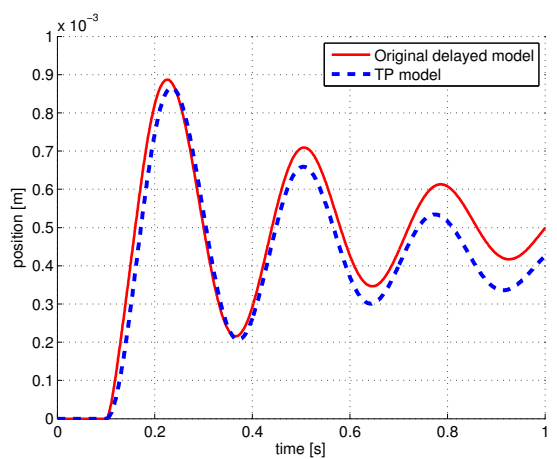

(d) Canonical form with 3 vertices

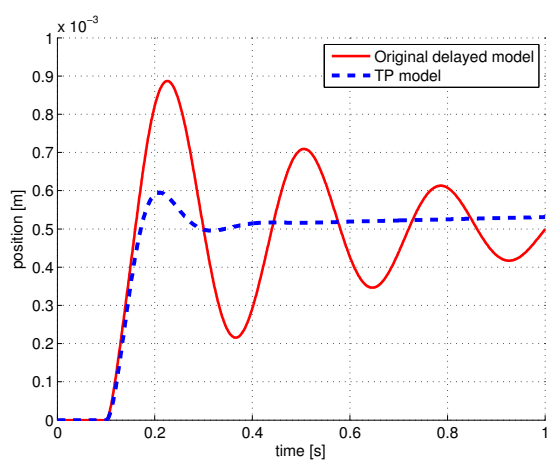

(f) Canonical form with 1 vertices

Figure 6

Comparison of the original delayed model and the HOSVD-based canonical form of the TP model with different complexity 


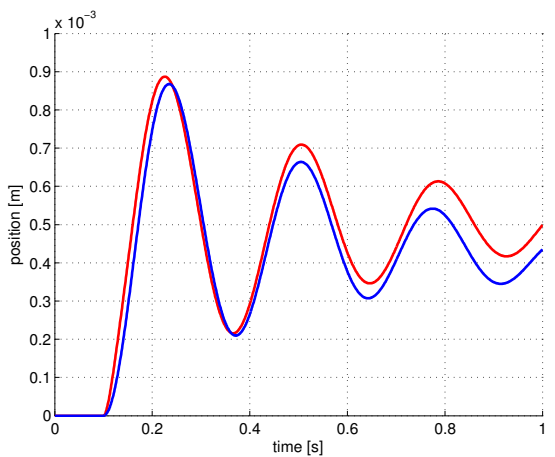

(a) $\mathrm{CNO5}$

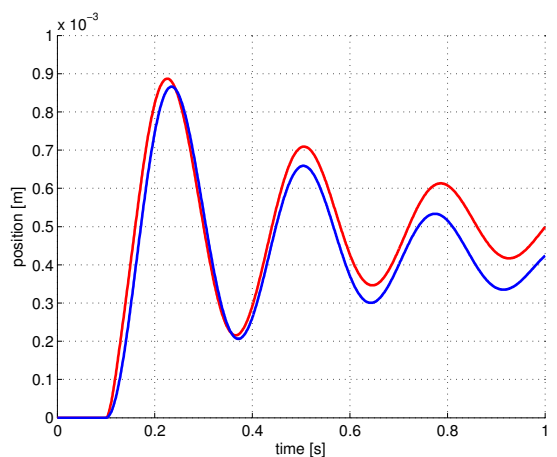

(b) $\mathrm{CNO} 4$

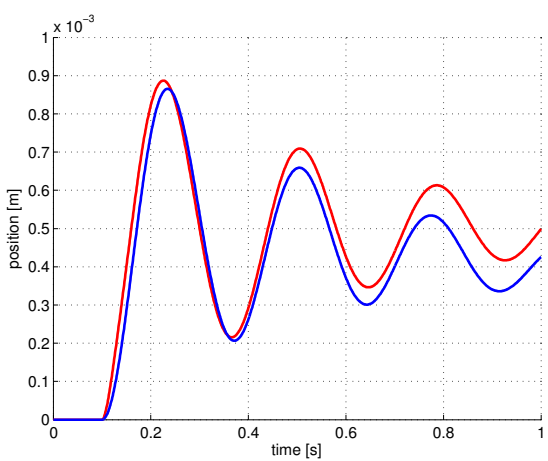

(c) $\mathrm{CNO} 3$

Figure 7

Comparison of the original delayed model and the CNO type TP model with different complexity

Table 1

Quantitative comparison of the original delayed model and the CNO type TP model with 3 vertices

\begin{tabular}{ccc}
\hline & $\mathcal{L}_{2}$ error & Max error \\
\hline$\tau=0.01375 s$ & $2.6279 \times 10^{-5}$ & $9.8521 \times 10^{-7}$ \\
$\tau=0.02941 s$ & $4.0380 \times 10^{-5}$ & $5.9765 \times 10^{-6}$ \\
$\tau=0.04752 s$ & $4.3281 \times 10^{-5}$ & $1.0500 \times 10^{-5}$ \\
$\tau=0.06393 s$ & $1.0851 \times 10^{-4}$ & $1.3048 \times 10^{-5}$ \\
\hline
\end{tabular}




\subsection{Varying time-delay}

The models have been compared under varying delay as well. The value of $\tau(t)$ was varied as a sine function of time $\tau(t)=0.03+\sin (t \pi) 0.025$. The input signal was a square wave with the frequency of $2 \mathrm{~Hz}$ and amplitude of $1 \mathrm{~N}$. Figure 8 shows the result of the simulation.
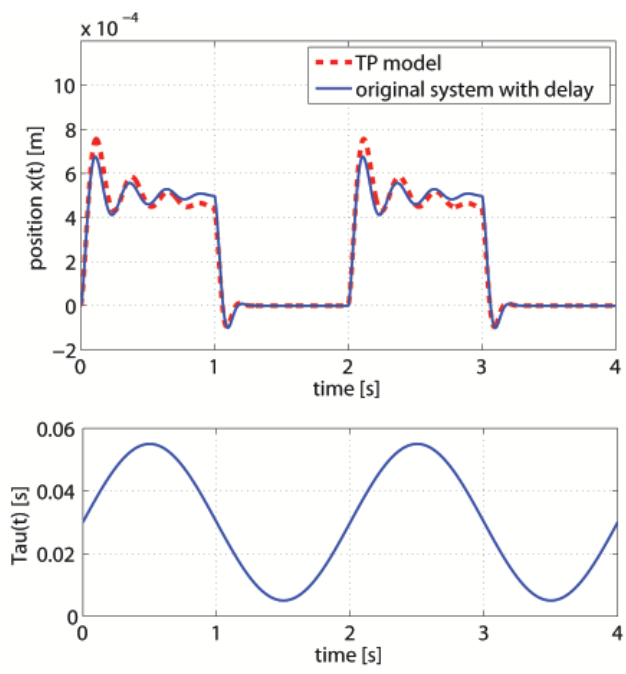

Figure 8

Comparison under varying delay

\section{Conclusion}

In this paper the HOSVD based canonical form of the model of the generic impedance controlled actuation with feedback delay was determined via a TP model transformation. A complexity trade off was also performed to determine non-exact TP models neglecting vertex systems with the less contribution. Via this investigation it has been proved that the TP model transformation is capable of manipulating the convex hull of the model wherein time delay $\tau$ appears as an external parameter. It has been shown that a convex polytop structure requires 6 vertex models for exact representation of the investigated model for any $\tau \in \Omega$. We presented the correlation between the number of vertex models and the number of singular values of the HOSVD based canonical form and the $L_{2}$ norm based error of the polytopic structure over the transformation space $\Omega$. In order to satisfy the basic requirements of LMI based design and further convex hull manipulation based optimization of the control design SNNN, IRNO and CNO type convex TP models was generated for the reduced 3 vertex model based convex hull. 


\section{Acknowledgement}

The research was supported by the Hungarian National Development Agency, (ERCHU-09-1-2009-0004 MTASZTAK) (OMFB-01677/2009).

\section{References}

[1] P. Baranyi, "TP model transformation as a way to LMI based controller design," IEEE Transaction on Industrial Electronics, vol. 51, pp. 387-400, April 2004.

[2] P. Baranyi, "Tensor-product model-based control of two-dimensional aeroelastic system," Journal of Guidance, Control, and Dynamics, vol. 29, pp. 391-400, May-June 2005.

[3] P. Baranyi, "Output feedback control of two-dimensional aeroelastic system," Journal of Guidance, Control, and Dynamics, vol. 29, pp. 762-767, May-June 2005 .

[4] F. Kolonic, A. Poljugan, and I. Petrovic, "Tensor product model transformationbased controller design for gantry crane control system - an application approach," Acta Polytechnica Hungarica, vol. 3, no. 4, pp. 95-112, 2006.

[5] R. Precup, L. Dioanca, E. M. Petriu, M. Radac, S. Preitl, and C. Dragos, "Tensor product-based real-time control of the liquid levels in a three tank system," in 2010 IEEE/ASME International Conference on Advanced Intelligent Mechatronics, (Montreal, QC, Canada), pp. 768-773, July 2010.

[6] Z. Szabó, P. Gáspár, and J. Bokor, "A novel control-oriented multi-affine qLPV modeling framework," in Control Automation (MED), 2010 18th Mediterranean Conference on, pp. 1019-1024, June 2010.

[7] S. Ilea, J. Matusko, and F. Kolonic, "Tensor product transformation based speed control of permanent magnet synchronous motor drives," in 17th International Conference on Electrical Drives and Power Electronics, EDPE 2011 (5th Joint Slovak-Croatian Conference), 2011.

[8] C. Sun, Y. Huang, C. Qian, and L. Wang, "On modeling and control of a flexible air-breathing hypersonic vehicle based on LPV method," Frontiers of Electrical and Electronic Engineering, vol. 7, no. 1, pp. 56-68, 2012.

[9] T. Luspay, T. Péni, and B. Kulcsar, "Constrained freeway traffic control via linear parameter varying paradigms," Control of Linear Parameter Varying Systems With Applications, p. 461, 2012.

[10] B. Takarics and P. Baranyi, "Friction compensation in TP model form - aeroelastic wing as an example system," Acta Polytechnica Hungarica, (Submitted).

[11] S. Chumalee and J. Whidborne, LPV Autopilot Design of a Jindivik UAV. American Institute of Aeronautics and Astronautics, 1801 Alexander Bell Dr., Suite 500 Reston VA 20191-4344 USA,, 2009. 
[12] S. Chumalee, Robust gain-scheduled $\mathcal{H}_{\infty}$ control for unmanned aerial vehicles. $\mathrm{PhD}$ thesis, Cranfield University, Cranfield, UK, 2010.

[13] W. Qin, Z. Zheng, G. Liu, J. Ma, and W. Li, "Robust variable gain control for hypersonic vehicles based on LPV," Systems Engineering and Electronics, vol. 33, no. 6, pp. 1327-1331, 2011.

[14] S. Rangajeeva and J. Whidborne, "Linear parameter varying control of a quadrotor," in Industrial and Information Systems (ICIIS), 2011 6th IEEE International Conference on, pp. 483-488, Aug. 2011.

[15] W. Gai, H. Wang, T. Guo, and D. Li, "Modeling and LPV flight control of the canard rotor/ wing unmanned aerial vehicle," in Artificial Intelligence, Management Science and Electronic Commerce (AIMSEC), 2011 2nd International Conference on, pp. 2187-2191, Aug. 2011.

[16] R. Precup, C. Dragos, S. Preitl, M. Radac, and E. M. Petriu, "Novel tensor product models for automatic transmission system control," IEEE Systems Journal, p. In print., 2012.

[17] P. Baranyi, "Convex hull generation methods for polytopic representations of LPV models," in Applied Machine Intelligence and Informatics, 2009. SAMI 2009. 7th International Symposium on, pp. 69-74, IEEE, 2009.

[18] P. Gróf, P. Baranyi, and P. Korondi, "Convex hull manipulation based control performance optimisation," WSEAS Transactions on Systems and Control, vol. 5, pp. 691-700, August 2010.

[19] L. D. Lathauwer, B. D. Moor, and J. Vandewalle, "A multilinear singular value decomposition," SIAM Journal on Matrix Analysis and Applications, vol. 21, no. 4, pp. 1253-1278, 2000.

[20] P. Baranyi, L. Szeidl, P. Várlaki, and Y. Yam, "Definition of the HOSVD-based canonical form of polytopic dynamic models," in 3rd International Conference on Mechatronics (ICM 2006), (Budapest, Hungary), pp. 660-665, July 3-5 2006.

[21] L. Szeidl and P. Várlaki, "HOSVD based canonical form for polytopic models of dynamic systems," Journal of Advanced Computational Intelligence and Intelligent Informatics, vol. 13, no. 1, pp. 52-60, 2009.

[22] N. Hogan, "Impedance control: An approach to manipulation: Part I-Theory," Journal of Dynamic Systems, Measurement, and Control, vol. 107, pp. 1-7, Mar. 1985.

[23] N. Hogan, "Impedance control: An approach to manipulation: Part IIImplementation," Journal of Dynamic Systems, Measurement, and Control, vol. 107, pp. 8-16, Mar. 1985. 
[24] N. Hogan, "Impedance control: An approach to manipulation: Part IIIApplications," Journal of Dynamic Systems, Measurement, and Control, vol. 107, pp. 17-24, Mar. 1985.

[25] T. Hulin, C. Preusche, and G. Hirzinger, "Stability boundary for haptic rendering: Influence of physical damping," in Intelligent Robots and Systems, 2006 IEEE/RSJ International Conference on, pp. 1570-1575, 2006.

[26] T. Hulin, C. Preusche, and G. Hirzinger, "Stability boundary for haptic rendering: Influence of human operator," in Intelligent Robots and Systems, 2008. IROS 2008. IEEE/RSJ International Conference on, pp. 3483-3488, 2008.

[27] J. J. Gil, E. Sanchez, T. Hulin, C. Preusche, and G. Hirzinger, "Stability boundary for haptic rendering: Influence of damping and delay," Journal of Computing and Information Science in Engineering, vol. 9, pp. 011005-8, Mar. 2009.

[28] S. H. Kang, M. Jin, and P. H. Chang, "A solution to the Accuracy/Robustness dilemma in impedance control," Mechatronics, IEEE/ASME Transactions on, vol. 14, no. 3, pp. 282-294, 2009.

[29] P. Galambos, P. Baranyi, and P. Korondi, "Extended TP model transformation for polytopic representation of impedance model with feedback delay," WSEAS Transactions on Systems and Control, vol. 5, no. 9, pp. 701-710, 2010.

[30] E. Biglieri and K. Yao, "Some properties of singular value decomposition and their applications to digital signal processing," Signal Processing, vol. 18, no. 3, pp. $277-289,1989$.

[31] Y. Nesterov and A. Nemirovskii, Interior-Point Polynomial Algorithms in Programming. Philadelphia: SIAM, 1994. 